\title{
Application of the Convergence of the Control Net of Box Splines to Scale-Space Filtering
}

\author{
Sylvain Meignen* \\ LMC-IMAG Laboratory, University of Grenoble, France \\ Tel:0033-4-76-51-43-95 \\ FAX:0033-4-76-63-12-63 \\ E-mail: sylvain.meignen@imag.fr
}

\begin{abstract}
In this paper, we propose a new approach to scale-space filtering using a box spline representation of multidimensional signals. The use of box splines is motivated by their ability to better handle complex geometries than tensor-product B-splines. The box spline we use is defined by a set of vectors invariant under the multiplication by a sampling matrix. We show that such a box spline satisfies a dilation equation which is the basis for the scale-space filtering we propose. Several numerical applications in 2D conclude the paper.
\end{abstract}

Keywords- Scale-space filtering, control net of box splines EDICS Category:WAVL,FBNK 


\section{INTRODUCTION}

Scale-space filtering traditionally consisted in an embedding of a signal into a one-parameter family of derived signal constructed by convolution with Gaussian kernels of increasing width. Neurophysiological research [16] have shown that primary visual cortex response can be modeled by the convolution with a superposition of Gaussian derivatives at increasing scales. In practice, when the scale gets larger, many techniques have been proposed for efficient implementation of scale-space filtering. Among them, Bsplines or binomials have been widely used to approximate the Gaussian kernel [13][15]. In that context, tensor-product B-splines naturally define bi-dimensional scale-space filterings. Tensor-product splines have been shown to be inappropriate for the modeling of complex objects in numerous applications due to their definition over arbitrary rectangular parameter domain [12]. An important class of functions that enables the definition of scale-space filtering without the disadvantages of tensor-product surfaces are box splines.

In this paper, we propose a new scale-space filtering based on a box spline representation of multidimensional signals. Having introduced the main definitions on box splines, we recall the equation they satisfy for a dilation by a factor of $m$ [2]. We then show how to write this equation when $m$ is replaced by an invertible sampling matrix $\mathbf{M}$ [11][8]. When the set of vectors that defines the box spline is invariant under multiplication by $\mathbf{M}$, we derive a similar equation to that of dilation. We, therefore, restrict ourselves to such box splines. We then define a sequence of piecewise constant functions that converges to the box spline under specific conditions. The construction of these piecewise constant functions is based on the convergence of the control net of box splines. Using this function and the property of the box spline with respect to the matrix $\mathbf{M}$, we are able to define a fast algorithm for multidimensional continuous scale-space filtering at rational scales and specific locations. This result is a generalization of that obtained on one dimensional scale-space filtering using a B-spline representation of signals [9].

The sketch of the article as follows. In section II, we recall the definition of box splines. Then, we highlight subdivision schemes for box splines. Finally, we review the convergence properties of the control net of box splines. Section III is devoted to the derivation of a new scale-space filtering. In section IV, we study, in 2D, the general form for the sampling matrix $\mathbf{M}$ and we put forward, in each case, a vector set invariant under multiplication by $\mathbf{M}$. Some numerical examples on the new scale-space filtering we propose conclude the paper. 


\section{Subdivision Schemes for Box Splines, Convergence of the Control Net of Box}

\section{SPLINES}

\section{A. Definition of Box Splines}

Let us define a set of $n$ vectors, not necessarily distinct, in an s-dimensional space

$$
\mathbf{X}_{n}=\left\{\mathbf{x}_{1}, \mathbf{x}_{2}, \cdots, \mathbf{x}_{n}\right\} \subset \mathbb{Z}^{s} \backslash\{0\}
$$

We assume that at least $s$ vectors of $\mathbf{X}_{n}$ are linearly independent. Let us rearrange the family $\mathbf{X}_{n}$ such that $\mathbf{X}_{s}=\left\{\mathbf{x}_{1}, \cdots, \mathbf{x}_{s}\right\}$ are linearly independent. Using the notation $\left[\mathbf{x}_{1}, \cdots, \mathbf{x}_{s}\right]\left[0,1\left[^{s}\right.\right.$ to denote the collection of linear combinations $\sum_{i=1}^{s} \lambda_{i} \mathbf{x}_{i}$ with $\lambda_{i} \in[0,1$, we define multivariate box splines as follows [3][10]:

$$
\begin{aligned}
& \beta\left(\mathbf{x}, \mathbf{X}_{s}\right)=\left\{\begin{aligned}
& \frac{1}{\left|\operatorname{det}\left(\mathbf{x}_{1}, \cdots, \mathbf{x}_{s}\right)\right|} \text { if } \mathbf{x} \in\left[\mathbf{x}_{1}, \cdots, \mathbf{x}_{s}\right]\left[0,1\left[^{s}\right.\right. \\
& 0 \text { otherwise }
\end{aligned}\right. \\
& \beta\left(\mathbf{x}, \mathbf{X}_{k}\right)=\int_{0}^{1} \beta\left(\mathbf{x}-t \mathbf{x}_{k}, \mathbf{X}_{k-1}\right) d t, \quad n \geq k>s .
\end{aligned}
$$

One can check by induction that the support of $\beta\left(\mathbf{x}, \mathbf{X}_{n}\right)$ is $\left[\mathbf{x}_{1}, \mathbf{x}_{2}, \cdots, \mathbf{x}_{n}\right][0,1]^{n}$.

\section{B. Dilation Equation for Box Splines}

The dilation equation satisfied by discrete box splines, defined as in (1), was introduced in [2]. It is proved that $\beta$ satisfies the relation:

$$
\beta\left(\frac{\mathbf{k}}{m}, \mathbf{X}_{n}\right)=\sum_{\mathbf{p} \in \mathbb{Z}^{s}} b_{m}\left[\mathbf{p}, \mathbf{X}_{n}\right] \beta\left(\mathbf{k}-\mathbf{p}, \mathbf{X}_{n}\right) \quad \forall \mathbf{k} \in \mathbb{Z}^{s}
$$

with

$$
b_{m}\left[\mathbf{p}, \mathbf{X}_{n}\right]=\frac{1}{m^{n-s}}\left(b_{m}\left[., \mathbf{x}_{1}\right] * \cdots * b_{m}\left[., \mathbf{x}_{n}\right]\right)[\mathbf{p}],
$$

where $b_{m}\left[., \mathbf{x}_{i}\right]$ is the succession of $m$ ones in the direction defined by $\mathbf{x}_{i}$ and $*$ denotes the $s$-dimensional convolution, that is $(f * g)[\mathbf{q}]=\sum_{\mathbf{l} \in \mathbb{Z}^{s}} f[\mathbf{l}] g[\mathbf{q}-\mathbf{l}]$. Equation (2) is proved using the $\mathbf{z}$-transform of $\beta\left(\frac{\mathbf{k}}{m}, \mathbf{X}_{n}\right)$. A more general result holds for all $\mathrm{x} \in \mathbb{R}^{\mathrm{s}}$ :

$$
\beta\left(\mathbf{x}, \mathbf{X}_{n}\right)=\sum_{\mathbf{p} \in \mathbb{Z}^{s}} b_{m}\left[\mathbf{p}, \mathbf{X}_{n}\right] \beta\left(m \mathbf{x}-\mathbf{p}, \mathbf{X}_{n}\right) \quad \forall \mathbf{x} \in \mathbb{R}^{s},
$$

which we do not prove since it is a particular case of what we deal with next, taking $\mathbf{M}=m \mathbf{I}_{s}$, where $\mathbf{I}_{s}$ is the identity matrix of size $s \times s$ (see the definition of $\mathbf{M}$ in the following). In case we consider a centered box spline, that is $\beta_{c}\left(\mathbf{x}, \mathbf{X}_{n}\right)=\beta\left(\mathbf{x}-\frac{1}{2}\left(\mathbf{x}_{1}+\cdots+\mathbf{x}_{n}\right), \mathbf{X}_{n}\right)$, relation (3) leads to $\beta_{c}(\mathbf{x})=\sum_{\mathbf{p} \in \mathbb{Z}^{s}} b_{m}\left[\mathbf{p}, \mathbf{X}_{n}\right] \beta_{c}\left(m \mathbf{x}-\frac{m-1}{2}\left(\mathbf{x}_{1}+\cdots+\mathbf{x}_{n}\right)-\mathbf{p}\right)$. For the sake of simplicity, we will maintain the definition (1) of box splines. 


\section{Non-Separable Dilation Equation for Box Splines}

In a more general framework, the dilation factor $m$ can be replaced by an invertible matrix $\mathbf{M}$ of integer entries, having real eigenvalues of modulus strictly larger than 1[8]. The matrix $\mathbf{M}$ must also be such that there exists a permutation $\sigma$ of $\{1, \cdots, n\}$ satisfying

$$
\mathbf{M} \mathbf{x}_{p}=\lambda_{p} \mathbf{x}_{\sigma(p)}
$$

with $\lambda_{p}$ a positive integer. When property (4) is satisfied, $\mathbf{X}_{n}$ is said to be invariant under multiplication by $\mathbf{M}$. The definition of $\sigma$ imposes that whenever two distinct vectors are in $\mathbf{X}_{n}$, each of them appears the same number of times in $\mathbf{X}_{n}$. Let us now state the non-separable dilation equation for box splines.

Theorem 1: Assume that $\mathbf{M}$ satisfies hypothesis (4) and that $\beta$ is defined as in (1) then,

$$
\beta\left(\mathbf{x}, \mathbf{X}_{n}\right)=\sqrt{|\operatorname{det}(\mathbf{M})|} \sum_{\mathbf{p} \in \mathbb{Z}^{s}} g\left[\mathbf{p}, \Lambda_{n}, \mathbf{X}_{\sigma(n)}\right] \beta\left(\mathbf{M x}-\mathbf{p}, \mathbf{X}_{n}\right),
$$

where $g\left[., \Lambda_{n}, \mathbf{X}_{n}\right]$ and $\Lambda_{n}=\left(\lambda_{1}, \cdots, \lambda_{n}\right)$, is defined by:

$$
g\left[\mathbf{p}, \Lambda_{n}, \mathbf{X}_{n}\right]=\frac{\sqrt{|\operatorname{det}(\mathbf{M})|}}{\prod_{p=1}^{n} \lambda_{p}}\left(b_{\lambda_{1}}\left[., \mathbf{x}_{1}\right] * \cdots * b_{\lambda_{n}}\left[., \mathbf{x}_{n}\right]\right)[\mathbf{p}]
$$

The proof is given in Appendix A. Taking $\mathbf{M}=m \mathbf{I}_{s}$, we find equation (3). Going further, we can replace $\mathbf{M}$ by $\mathbf{M}^{m}$, leading to another non-separable dilation equation.

Theorem 2: Assuming the same hypotheses as for Theorem 1, we have:

$$
\beta\left(\mathbf{x}, \mathbf{X}_{n}\right)=\sqrt{|\operatorname{det}(\mathbf{M})|^{m}} \sum_{\mathbf{p} \in \mathbb{Z}^{s}} g_{m}\left[\mathbf{p}, \Lambda_{n}, \mathbf{X}_{n}\right] \beta\left(\mathbf{M}^{m} \mathbf{x}-\mathbf{p}, \mathbf{X}_{n}\right),
$$

with

$$
g_{m}\left[\mathbf{p}, \Lambda_{n}, \mathbf{X}_{n}\right]=\left(\tilde{g}\left[., \Lambda_{n}, \mathbf{X}_{\sigma(n)}\right] * \cdots * \tilde{g}\left[., \Lambda_{n}, \mathbf{X}_{\sigma^{m}(n)}\right]\right)[\mathbf{p}],
$$

where

$$
\tilde{g}\left[\mathbf{p}, \Lambda_{n}, \mathbf{X}_{\sigma(n)}\right]=g\left[\mathbf{p}, \Lambda_{n}, \mathbf{X}_{\sigma(n)}\right]
$$

and for $r \geq 2$,

$$
\tilde{g}\left[\mathbf{p}, \Lambda_{n}, \mathbf{X}_{\sigma^{r}(n)}\right]=\frac{\sqrt{|\operatorname{det}(\mathbf{M})|}}{\prod_{p=1}^{n} \lambda_{p}}\left(\left(b_{\lambda_{1}}\right)_{\uparrow \prod_{k=1}^{r-1} \lambda_{\sigma^{k}(1)}}\left[., \mathbf{x}_{\sigma^{r}(1)}\right] * \cdots *\left(b_{\lambda_{n}}\right) \uparrow_{\uparrow_{k=1}^{r-1} \lambda_{\sigma^{k}(n)}}\left[., \mathbf{x}_{\sigma^{r}(n)}\right]\right)[\mathbf{p}] .
$$

The proof is given in Appendix B. 


\section{Convergence of the Control Net of Box Splines}

From now on, we assume that $\left\{\mathbf{x}_{1}, \cdots, \mathbf{x}_{j}^{*}, \cdots, \mathbf{x}_{n}\right\}$ spans $\mathbb{R}^{s}$ for all $j \in\{1, \cdots, n\}$, where $\mathbf{x}_{j}^{*}$ means we have removed $\mathbf{x}_{j}$ from $\mathbf{X}_{n}$. In the following, we consider a box spline defined as in (1) but the results are also true for a centered box spline, applying appropriate shifts. With the above hypothesis on $\mathbf{X}_{n}$, $\beta\left(\mathbf{x}, \mathbf{X}_{n}\right)$ is continuous and the span of its shifts contains linear polynomials [10]. In particular, if we put

$$
\mathbf{n}_{\mathbf{p}}=\mathbf{p}+\frac{1}{2}\left(\mathbf{x}_{1}+\cdots+\mathbf{x}_{n}\right), \quad \mathbf{p} \in \mathbb{Z}^{s},
$$

we have $\sum_{\mathbf{p} \in \mathbb{Z}^{s}} \mathbf{n}_{\mathbf{p}} \beta\left(\mathbf{x}-\mathbf{p}, \mathbf{X}_{n}\right)=\mathbf{x}$. Indeed, if we denote $\mathbf{n}_{\mathbf{0}}=\frac{1}{2}\left(\mathbf{x}_{1}+\cdots+\mathbf{x}_{n}\right)$ the center of the box spline $\beta$ then,

$$
\begin{aligned}
\sum_{\mathbf{p} \in \mathbb{Z}^{s}} \mathbf{n}_{\mathbf{p}} \beta\left(\mathbf{n}_{\mathbf{0}}-\mathbf{p}, \mathbf{X}_{n}\right) & =\mathbf{n}_{\mathbf{0}} \sum_{\mathbf{p} \in \mathbb{Z}^{s}} \beta\left(\mathbf{n}_{\mathbf{0}}-\mathbf{p}, \mathbf{X}_{n}\right)+\sum_{\mathbf{p} \in \mathbb{Z}^{s}} \mathbf{p} \beta\left(\mathbf{n}_{\mathbf{0}}-\mathbf{p}, \mathbf{X}_{n}\right) \\
& =\mathbf{n}_{\mathbf{0}},
\end{aligned}
$$

since $\sum_{\mathbf{p} \in \mathbb{Z}^{s}} \beta\left(\mathbf{n}_{\mathbf{0}}-\mathbf{p}, \mathbf{X}_{n}\right)=1[10]$ and $\beta\left(\mathbf{n}_{\mathbf{0}}-\mathbf{p}, \mathbf{X}_{n}\right)=\beta\left(\mathbf{n}_{\mathbf{0}}+\mathbf{p}, \mathbf{X}_{n}\right)$ (the box spline $\beta$ being symmetric with respect to its center). Now, if we denote $D_{\mathbf{x}_{k}}$ the directional derivative in the direction $\mathbf{x}_{k}$, a simple computation leads to the equality $D_{\mathbf{x}_{k}}\left(\sum_{\mathbf{p} \in \mathbb{Z}^{s}} \mathbf{n}_{\mathbf{p}} \beta\left(\mathbf{x}-\mathbf{p}, \mathbf{X}_{n}\right)\right)=\mathbf{x}_{k}$, which states the expected result.

$$
\begin{aligned}
& \text { Now, if we define } \mathbf{B}\left(\mathbf{x}, \mathbf{X}_{n}\right)=\left(\begin{array}{c}
\mathbf{x} \\
\beta\left(\mathbf{x}, \mathbf{X}_{n}\right)
\end{array}\right) \text { and } \mathbf{c}_{m}^{\mathbf{p}}=\left(\begin{array}{c}
\frac{\mathbf{n}_{\mathbf{p}}}{m} \\
b_{m}\left[\mathbf{p}, \mathbf{X}_{n}\right]
\end{array}\right) \text {, we can write: } \\
& \qquad \mathbf{B}\left(\mathbf{x}, \mathbf{X}_{n}\right)=\sum_{\mathbf{p} \in \mathbb{Z}^{s}} \mathbf{c}_{m}^{\mathbf{p}} \beta\left(m \mathbf{x}-\mathbf{p}, \mathbf{X}_{n}\right) .
\end{aligned}
$$

It was proved by De Boor et al. [6], that if $\left[\mathbf{x}_{1}, \cdots, \mathbf{x}_{n}\right] \mathbb{Z}^{n}=\mathbb{Z}^{s}$, where $\left[\mathbf{x}_{1}, \cdots, \mathbf{x}_{n}\right] \mathbb{Z}^{n}$ denotes the collection of linear combinations with integer coefficients, then $\left\|\mathbf{c}_{m}^{\mathbf{p}}-\mathbf{B}\left(\mathbf{x}, \mathbf{X}_{n}\right)\right\|=O\left(\frac{1}{m}\right)$, for any $\mathbf{x}$ such that $\beta\left(m \mathbf{x}-\mathbf{p}, \mathbf{X}_{n}\right) \geq 0$. This equality implies in particular that:

$$
\left|b_{m}\left[\mathbf{p}, \mathbf{X}_{n}\right]-\beta\left(\mathbf{x}, \mathbf{X}_{n}\right)\right|=O\left(\frac{1}{m}\right) .
$$

If the box spline is continuously differentiable, then the approximation is in $O\left(\frac{1}{m^{2}}\right)$.

\section{Piecewise Constant Approximation of $\beta$ And Multidimensional Scale-Space}

\section{FILTERING}

\section{A. Definition of a Piecewise Constant Approximation of $\beta$}

We now explain how to define a sequence of piecewise constant functions that uniformly converges to $\beta$ with $m$, where $m$ is the dilation factor of equation (3). We recall that the points $\frac{\mathbf{n}_{\mathbf{p}}}{m}$, where $\mathbf{n}_{\mathbf{p}}$ is 
defined in (6), lie on the grid $\frac{\mathbb{Z}^{s}}{m}$ translated by $\frac{1}{2 m}\left(\mathbf{x}_{1}+\cdots+\mathbf{x}_{n}\right)$. We therefore consider the piecewise constant function:

$$
F_{m}\left(\mathbf{x}, \mathbf{X}_{n}\right)=b_{m}\left[\mathbf{p}, \mathbf{X}_{n}\right] \forall \mathbf{x} \in \operatorname{supp}(\beta)
$$

with $\mathbf{p}=\underset{\mathbf{q}}{\operatorname{argmin}}\left(\left\|\mathbf{x}-\frac{\mathbf{n}_{\mathbf{q}}}{m}\right\|_{\infty}\right)$ and where $\|\mathbf{x}\|_{\infty}=\max _{i}\left|x_{i}\right|$. Conversely, given $\mathbf{p}$, the set of points $\mathbf{x}$ satisfying this property is $V_{\mathbf{p}}^{m}=\left\{\mathbf{x},\left\|\mathbf{x}-\frac{\mathbf{n}_{\mathbf{p}}}{m}\right\|_{\infty}<\frac{1}{2 m}\right\}$. Similarly, we define

$$
V_{\mathbf{p}}=\left\{\mathbf{x},\left\|\mathbf{x}-\mathbf{n}_{\mathbf{p}}\right\|_{\infty}<\frac{1}{2}\right\} .
$$

We now give a simple condition for $F_{m}$ to converge uniformly to $\beta$.

Theorem 3: If we assume that $\beta$ is continuously differentiable, then $\left|F_{m}\left(\mathbf{x}, \mathbf{X}_{n}\right)-\beta\left(\mathbf{x}, \mathbf{X}_{n}\right)\right|=O\left(\frac{1}{m}\right)$. Proof: Any $\mathbf{x}$ in $\operatorname{supp}(\beta)$ belongs to $V_{\mathbf{p}}^{m}$ for some $\mathbf{p}$. A first order Taylor expansion at $\frac{\mathbf{n}_{\mathbf{p}}}{m}$ leads to:

$$
\begin{aligned}
\beta\left(\mathbf{x}, \mathbf{X}_{n}\right) & =\beta\left(\frac{\mathbf{n}_{\mathbf{p}}}{m}, \mathbf{X}_{n}\right)+O\left(\frac{1}{m}\right) \\
& =b_{m}\left[\mathbf{p}, \mathbf{X}_{n}\right]+O\left(\frac{1}{m}\right) \text { with (7) } \\
& =F_{m}\left(\mathbf{x}, \mathbf{X}_{n}\right)+O\left(\frac{1}{m}\right) \text { with (8). }
\end{aligned}
$$

\section{B. Multidimensional Scale-Space Filtering}

The linear scale-space representation is to make a map of a signal by changing the scale parameter continuously. In the language of wavelet transform, the traditional scale-space approach can be regarded as a continuous wavelet transform of the signal $f \in L^{2}\left(\mathbb{R}^{s}\right)$,

$$
W f(a, \mathbf{x})=\int_{\mathbb{R}^{s}} f(\mathbf{t}) \frac{1}{a^{s}} \Psi\left(\frac{\mathbf{t}-\mathbf{x}}{a}\right) d \mathbf{t}=\int_{\mathbb{R}^{s}} f(\mathbf{x}+\mathbf{t}) \frac{1}{a^{s}} \Psi\left(\frac{\mathbf{t}}{a}\right) d \mathbf{t} a>0 .
$$

In a box spline framework, we consider a wavelet $\Psi$ defined by

$$
\Psi(\mathbf{x})=\sqrt{|\operatorname{det}(\mathbf{M})|^{m}} \sum_{\mathbf{p} \in \mathbb{Z}^{s}} \alpha_{m}\left[\mathbf{p}, \mathbf{X}_{n}\right] \beta\left(\mathbf{M}^{m} \mathbf{x}-\mathbf{p}, \mathbf{X}_{n}\right),
$$

and a multidimensional signal $f$ approximated by

$$
f(\mathbf{x}) \approx \tilde{f}(\mathbf{x})=\sqrt{|\operatorname{det}(\mathbf{M})|^{m}} \sum_{\mathbf{p} \in \mathbb{Z}^{s}} \gamma_{m}\left[\mathbf{p}, \mathbf{X}_{n}\right] \beta\left(\mathbf{M}^{m} \mathbf{x}-\mathbf{p}, \mathbf{X}_{n}\right)
$$

To decompose the signal $f$ in such a way is interesting in that the use of non-diagonal sampling matrices such as quincunx or hexagonal sampling matrices is known to lead to superior results in signal and image coding [8]. The way the sequence $\gamma_{m}$ is computed is discussed in the next subsection. 
Since a real number can be approximated arbitrarily close by a rational number $a=\frac{m_{1}}{m_{2}}$, using (11) and (14), we derive:

$$
\begin{aligned}
W \tilde{f}\left(\frac{m_{1}}{m_{2}}, \mathbf{x}\right)= & \left|\operatorname{det}(\mathbf{M})^{m}\right|\left(\frac{m_{2}}{m_{1}}\right)^{s} \\
& \sum_{(\mathbf{p}, \mathbf{q}) \in\left(\mathbb{Z}^{s}\right)^{2}} \alpha_{m}\left[\mathbf{p}, \mathbf{X}_{n}\right] \gamma_{m}\left[\mathbf{q}, \mathbf{X}_{n}\right] \int_{\mathbb{R}^{s}} \beta\left(\frac{m_{2}}{m_{1}} \mathbf{M}^{m} \mathbf{t}-\mathbf{p}, \mathbf{X}_{n}\right) \beta\left(\mathbf{M}^{m}(\mathbf{x}+\mathbf{t})-\mathbf{q}, \mathbf{X}_{n}\right) d \mathbf{t} \\
= & \left(\frac{m_{2}}{m_{1}}\right)^{s} \sum_{(\mathbf{p}, \mathbf{q}) \in\left(\mathbb{Z}^{s}\right)^{2}} \alpha_{m}\left[\mathbf{p}, \mathbf{X}_{n}\right] \gamma_{m}\left[\mathbf{q}, \mathbf{X}_{n}\right] \int_{\mathbb{R}^{s}} \beta\left(\frac{m_{2}}{m_{1}} \mathbf{t}-\mathbf{p}, \mathbf{X}_{n}\right) \beta\left(\mathbf{M}^{m} \mathbf{x}+\mathbf{t}-\mathbf{q}, \mathbf{X}_{n}\right) d \mathbf{t} \\
= & \left(\frac{1}{m_{1}}\right)^{s} \sum_{(\mathbf{p}, \mathbf{q}, \mathbf{k}, \mathbf{l}) \in\left(\mathbb{Z}^{s}\right)^{4}} \alpha_{m}\left[\mathbf{p}, \mathbf{X}_{n}\right] \gamma_{m}\left[\mathbf{q}, \mathbf{X}_{n}\right] b_{m_{1}}\left[\mathbf{k}, \mathbf{X}_{n}\right] b_{m_{2}}\left[\mathbf{l}, \mathbf{X}_{n}\right] \\
& (\beta \tilde{*} \bar{\beta})\left(-m_{2} \mathbf{M}^{m} \mathbf{x}-m_{1} \mathbf{p}+m_{2} \mathbf{q}-\mathbf{k}+\mathbf{l}, \mathbf{X}_{n}\right),
\end{aligned}
$$

where $\bar{\beta}\left(\mathbf{x}, \mathbf{X}_{n}\right)=\beta\left(-\mathbf{x}, \mathbf{X}_{n}\right)$ and $\beta \tilde{*} \bar{\beta}\left(\mathbf{x}, \mathbf{X}_{n}\right)=\int_{\mathbb{R}^{s}} \beta\left(\mathbf{t}, \mathbf{X}_{n}\right) \bar{\beta}\left(\mathbf{x}-\mathbf{t}, \mathbf{X}_{n}\right) d \mathbf{t}$. One notices that $\beta \tilde{*} \bar{\beta}\left(\mathbf{x}, \mathbf{X}_{n}\right)=$ $\beta\left(\mathbf{x}, \tilde{\mathbf{X}}_{n}\right)$ with $\tilde{\mathbf{X}}_{n}=\left[\mathbf{x}_{1},-\mathbf{x}_{1}, \cdots, \mathbf{x}_{n},-\mathbf{x}_{n}\right]$ and is therefore symmetric with respect to zero. Then, taking $\mathbf{x}=\frac{\mathbf{M}^{-m_{\mathbf{i}}}}{m_{2}}$ and putting $\tilde{\beta}_{d}[\mathbf{i}]=\beta\left(\mathbf{i}, \tilde{\mathbf{X}}_{\mathbf{n}}\right)$ leads to:

$$
\begin{aligned}
W \tilde{f}\left(\frac{m_{1}}{m_{2}}, \frac{\mathbf{M}^{-m_{\mathbf{i}}}}{m_{2}}\right) & =\left(\frac{1}{m_{1}}\right)^{s} \sum_{(\mathbf{k}, \mathbf{l}) \in\left(\mathbb{Z}^{s}\right)^{2}}\left(\left(\gamma_{m}\right)_{\uparrow m_{2}} * b_{m_{2}}\right)\left[\mathbf{l}, \mathbf{X}_{n}\right] \overline{\left(\left(\alpha_{m}\right)_{\uparrow m_{1}} * b_{m_{1}}\right)}\left[\mathbf{k}, \mathbf{X}_{n}\right] \tilde{\beta}_{d}[\mathbf{i}-\mathbf{l}-\mathbf{k}] \\
& =\left(\frac{1}{m_{1}}\right)^{s}\left(\left(\gamma_{m}\right)_{\uparrow m_{2}} * b_{m_{2}} * \overline{\left(\alpha_{m}\right)_{\uparrow m_{1}}} * \overline{b_{m_{1}}} * \tilde{\beta}_{d}\right)\left[\mathbf{i}, \mathbf{X}_{n}\right] .
\end{aligned}
$$

We now see how to use the piecewise constant function $F_{m}$ to avoid the convolution with $\tilde{\beta}_{d}$, thus saving computational time. As $F_{m}$ uniformly converges to $\beta$ when $m$ tends to $+\infty$, we can write the following approximation for large $m_{1}$ and $m_{2}$, for the case where $\frac{m_{1}}{m_{2}}$ is constant:

$W \tilde{f}\left(\frac{m_{1}}{m_{2}}, \mathbf{x}\right) \approx\left(\frac{m_{2}}{m_{1}}\right)^{s} \sum_{\left((\mathbf{p}, \mathbf{q}) \in\left(\mathbb{Z}^{s}\right)^{2}\right.} \alpha_{m}\left[\mathbf{p}, \mathbf{X}_{n}\right] \gamma_{m}\left[\mathbf{q}, \mathbf{X}_{n}\right] \int_{\mathbb{R}^{s}} F_{m_{1}}\left(\frac{m_{2}}{m_{1}} \mathbf{t}-\mathbf{p}, \mathbf{X}_{n}\right) F_{m_{2}}\left(\mathbf{M}^{m} \mathbf{x}+\mathbf{t}-\mathbf{q}, \mathbf{X}_{n}\right) d \mathbf{t}$.

Let us then rewrite formally the integral:

$$
\begin{array}{r}
\int_{\mathbb{R}^{s}} F_{m_{1}}\left(\frac{m_{2}}{m_{1}} \mathbf{t}-\mathbf{p}, \mathbf{X}_{n}\right) F_{m_{2}}\left(\mathbf{M}^{m} \mathbf{x}+\mathbf{t}-\mathbf{q}, \mathbf{X}_{n}\right) d \mathbf{t} \\
=\frac{1}{m_{2}^{s}} \sum_{\mathbf{r} \in \mathbb{Z}^{s}} \int_{V_{\mathbf{r}}} F_{m_{1}}\left(\frac{\mathbf{t}}{m_{1}}-\mathbf{p}, \mathbf{X}_{n}\right) F_{m_{2}}\left(\mathbf{M}^{m} \mathbf{x}+\frac{\mathbf{t}}{m_{2}}-\mathbf{q}, \mathbf{X}_{n}\right) d \mathbf{t} \text { using (9) } \\
=\frac{1}{m_{2}^{s}} \sum_{\mathbf{r} \in \mathbb{Z}^{s}} b_{m_{1}}\left[\mathbf{r}-m_{1} \mathbf{p}, \mathbf{X}_{n}\right] b_{m_{2}}\left[\mathbf{r}+m_{2}\left(\mathbf{M}^{m} \mathbf{x}-\mathbf{q}\right), \mathbf{X}_{n}\right]
\end{array}
$$

Consequently, if $\mathbf{x}=\frac{\mathbf{M}^{-m} \mathbf{i}}{m_{2}}$, with $\mathbf{i}$ in $\mathbb{Z}^{s}$, the above expression makes sense. We, finally, get the following 
approximation for the scale-space filtering:

$$
\begin{array}{r}
W \tilde{f}\left(\frac{m_{1}}{m_{2}}, \frac{\mathbf{M}^{-m_{i}} \mathbf{i}}{m_{2}}\right) \approx \frac{1}{m_{1}^{s}} \sum_{(\mathbf{p}, \mathbf{q}, \mathbf{r}) \in\left(\mathbb{Z}^{s}\right)^{3}} \alpha_{m}\left[\mathbf{p}, \mathbf{X}_{n}\right] \gamma_{m}\left[\mathbf{q}, \mathbf{X}_{n}\right] b_{m_{1}}\left[\mathbf{r}-m_{1} \mathbf{p}, \mathbf{X}_{n}\right] b_{m_{2}}\left[\mathbf{r}+\mathbf{i}-m_{2} \mathbf{q}, \mathbf{X}_{n}\right] \\
=\frac{1}{m_{1}^{s}} \sum_{\mathbf{r} \in \mathbb{Z}^{s}} \sum_{\mathbf{q} \in \mathbb{Z}^{s}} \gamma_{m}\left[\mathbf{q}, \mathbf{X}_{n}\right] b_{m_{2}}\left[\mathbf{r}+\mathbf{i}-m_{2} \mathbf{q}, \mathbf{X}_{n}\right] \sum_{\mathbf{p} \in \mathbb{Z}^{s}} \alpha_{m}\left[\mathbf{p}, \mathbf{X}_{n}\right] b_{m_{1}}\left[\mathbf{r}-m_{1} \mathbf{p}, \mathbf{X}_{n}\right] \\
=\frac{1}{m_{1}^{s}} \sum_{\mathbf{r} \in \mathbb{Z}^{s}}\left(\left(\gamma_{m}\right)_{\uparrow m_{2}} * b_{m_{2}}\right)\left[\mathbf{i}+\mathbf{r}, \mathbf{X}_{n}\right]\left(\left(\alpha_{m}\right)_{\uparrow m_{1}} * b_{m_{1}}\right)\left[\mathbf{r}, \mathbf{X}_{n}\right] \\
=\frac{1}{m_{1}^{s}}\left(\left(\gamma_{m}\right)_{\uparrow m_{2}} * b_{m_{2}} * \overline{\left(\alpha_{m}\right)_{\uparrow m_{1}}} * \overline{b_{m_{1}}}\right)\left[\mathbf{i}, \mathbf{X}_{n}\right]=W \tilde{f}_{a}\left(\frac{m_{1}}{m_{2}}, \frac{\mathbf{M}^{-m_{i}}}{m_{2}}\right) .
\end{array}
$$

As expected, to compute $W \tilde{f}_{a}$ instead of $W \tilde{f}$ avoids the convolution by $\tilde{\beta}_{d}$. Furthermore, we will see, in the Numerical Applications section, why such a scale-space filtering based on the lattice $\left(\frac{\mathbf{M}^{-m_{\mathbf{i}}}}{m_{2}}\right)_{\mathbf{i} \in \mathbb{Z}^{s}}$ is interesting.

\section{Computation of $\alpha_{m}$ and $\gamma_{m}$}

Let us first set $\gamma=\gamma_{0}$. The sequence $\gamma$ cannot be obtained by interpolation as in the B-spline case [13] since, in most cases, the decomposition over the span of shifts of a box spline is not unique. The uniqueness is proved if and only if the matrix $\left[\mathbf{x}_{1}, \cdots, \mathbf{x}_{n}\right]$ is unimodular, that is if the determinant of any of its submatrices of size $s^{2}$ is 1 or -1 [4]. An approach to compute the coefficients of the decomposition over shifted box splines is proposed in [3]. It is based on a quasi-interpolation formulation of the problem. The interpolation problem is to find $\gamma$ such that $f(\mathbf{p})=\sum_{\mathbf{l} \in \mathbb{Z}^{s}} \gamma[\mathbf{l}] \beta\left(\mathbf{p}-\mathbf{l}, \mathbf{X}_{n}\right)$. Taking the $\mathbf{z}$-transform of both sides of this equality leads to $F(\mathbf{z})=\Gamma(\mathbf{z}) \Delta\left(\mathbf{z}, \mathbf{X}_{n}\right)$, where $\Delta$ is the z-transform of $\beta$. If we put $\Delta\left(\mathbf{z}, \mathbf{X}_{n}\right)^{-1}=\left(1-D\left(\mathbf{z}, \mathbf{X}_{n}\right)\right)^{-1}$, we have the following approximation of $\Gamma(\mathbf{z})$ for some $q$ : $\Gamma(\mathbf{z}) \approx F(\mathbf{z})\left(1+D\left(\mathbf{z}, \mathbf{X}_{n}\right)+\cdots+D^{q}\left(\mathbf{z}, \mathbf{X}_{n}\right)\right)$. The stability of the method requires that the roots of $\Delta\left(\mathbf{z}, \mathbf{X}_{n}\right)$ be inside the unit ball. This is, in practice, a very strong restriction. For instance, if one considers the most popular box spline based on $\mathbf{X}_{n}=\left\{\left[\begin{array}{ll}1 & 0\end{array}\right]^{T},\left[\begin{array}{ll}-1 & 1\end{array}\right]^{T},\left[\begin{array}{ll}0 & 1\end{array}\right]^{T},\left[\begin{array}{ll}1 & 1\end{array}\right]^{T}\right\}$, then we have

$$
\Delta\left(\mathbf{z}, \mathbf{X}_{n}\right)=\frac{1}{4} z_{2}^{-1}\left(1+z_{2}^{-1}+z_{1}^{-1}+z_{1}^{-1} z_{2}^{-1}\right)
$$

where $\mathbf{z}=\left(z_{1}, z_{2}\right)$. One sees that it is null for $\mathbf{z}=(1,-1)$ which is not inside the unit ball. Therefore, the algorithm proposed in [3] is instable in this case.

We adopt a mean square approach for the computation of $\gamma$. The advantage of such an approach is that it does not impose any condition on $\beta$. To compute $\gamma$, we first define

$$
E(\gamma)=\frac{1}{2} \sum_{\mathbf{k} \in \mathbb{Z}^{s}}\left(f(\mathbf{k})-\sum_{\mathbf{l} \in \mathbb{Z}^{2}} \gamma[\mathbf{l}] \beta\left(\mathbf{k}-\mathbf{l}, \mathbf{X}_{n}\right)\right)^{2}
$$


If we differentiate this expression with respect to $\gamma$, we obtain

$$
\partial_{\gamma} E[\mathbf{l}]=-\left(f * \bar{\beta}_{d}\right)[\mathbf{l}]+\left(\gamma * \beta_{d} * \bar{\beta}_{d}\right)[\mathbf{l}],
$$

where $\beta_{d}[\mathbf{l}]=\beta\left(\mathbf{l}, \mathbf{X}_{n}\right)$ and $\bar{\beta}_{d}[\mathbf{l}]=\beta_{d}[-\mathbf{l}]$. We then use a gradient descent method to compute $\gamma$. In such a framework, the value of $\gamma$ we obtain corresponds to a local minimum for $E$. In applications, we always check that the box spline approximation is close to the original image. In [1], a fast approach for the computation of $\gamma$ is proposed since, when initial condition is far from an optimum solution, the convergence of the gradient descent method may be slow.

Once $\gamma$ is computed, we use (5) to write:

$$
\begin{array}{r}
\tilde{f}(\mathbf{x})=\sum_{\mathbf{l} \in \mathbb{Z}^{s}} \gamma[\mathbf{l}] \beta\left(\mathbf{x}-\mathbf{l}, \mathbf{X}_{n}\right) \\
=\sqrt{|\operatorname{det}(\mathbf{M})|^{m}} \sum_{\mathbf{p} \in \mathbb{Z}^{s}}\left(g_{m}\left[., \Lambda_{n}, \mathbf{X}_{n}\right] * \gamma_{\uparrow \mathbf{M}^{m}}\right)[\mathbf{p}] \beta\left(\mathbf{M}^{m} \mathbf{x}-\mathbf{p}, \mathbf{X}_{n}\right),
\end{array}
$$

where

$$
\gamma_{\uparrow \mathbf{M}^{m}}[\mathbf{q}]=\left\{\begin{array}{c}
\gamma[\mathbf{p}] \text { if } \exists \mathbf{p} \in \mathbb{Z}^{s} \text { such that } \mathbf{q}=\mathbf{M}^{m} \mathbf{p} \\
0 \text { otherwise. }
\end{array}\right.
$$

This means that $\gamma_{m}=g_{m} * \gamma_{\uparrow \mathbf{M}^{m}}$.

The wavelets we consider are called box spline wavelets (for details on these wavelets see [12]) and are of the form:

$$
\Psi(\mathbf{x})=\sum_{\mathbf{p} \in \mathbb{Z}^{s}} \alpha[\mathbf{p}] \beta\left(\mathbf{x}-\mathbf{p}, \mathbf{X}_{n}\right),
$$

with $\sum_{\mathbf{p} \in \mathbb{Z}^{s}} \alpha[\mathbf{p}]=0$ where $\alpha$ is a finite sequence. Indeed, since $\int_{\mathbb{R}^{s}} \beta\left(\mathbf{x}, \mathbf{X}_{n}\right) d \mathbf{x}=1, \int_{\mathbb{R}^{s}} \Psi(\mathbf{x}) d \mathbf{x}=0$. To get a wavelet with a larger number of vanishing moments would require to impose stronger conditions on $\alpha$, but this is not the point here. Then, with (5), we deduce:

$$
\Psi(\mathbf{x})=\sqrt{|\operatorname{det}(\mathbf{M})|^{\mathbf{m}}} \sum_{\mathbf{p} \in \mathbb{Z}^{s}}\left(g_{m}\left[., \Lambda_{n}, \mathbf{X}_{n}\right] * \alpha_{\uparrow \mathbf{M}^{m}}\right)[\mathbf{q}] \beta\left(\mathbf{M}^{m} \mathbf{x}-\mathbf{q}, \mathbf{X}_{n}\right) .
$$

In all cases, $\alpha_{m}=g_{m} * \alpha_{\uparrow \mathbf{M}^{m}}$.

\section{Study of the Matrix M when $s=2$, Definition of Invariant Vector Sets}

\section{A. General Form for the Matrix $\mathbf{M}$}

We now investigate the case $s=2$ in detail and more precisely the influence of (4) on matrix $\mathbf{M}$, assuming, without any loss of generality, that $\mathbf{X}_{n}$ is composed of distinct vectors. We study the length of the cycles that make up the permutation $\sigma$. A cycle of length $q$ is defined by a subset $S$ of $\mathbf{X}_{n}$ such that 
$q$ is the smallest integer satisfying $\mathbf{M}^{q} \mathbf{x}_{i}=a_{i} \mathbf{x}_{i}=\lambda \mathbf{x}_{i}$ for all $\mathbf{x}_{i}$ in $S . a_{i}$ is independent from $i$ since $a_{i}=\prod_{i \in J_{S}} \lambda_{i}$, where $J_{S}$ is the set of indices corresponding to $S$. Since at least $s \mathbf{x}_{i}$ s' are non-collinear, the existence of a cycle of length $q \geq 2$ implies

$$
\mathbf{M}^{q}=\lambda \mathbf{I}_{2} .
$$

Assume there exists a cycle with $q \geq 3$ and $q$ odd. Since $\mathbf{M}$ is invertible, if the eigenvalues of $\mathbf{M}$ are $a$ and $b$ and if $\mathbf{u}$ and $\mathbf{v}$ are eigenvectors associated with $a$ and $b$ respectively, then $\mathbf{M}^{q} \mathbf{u}=a^{q} \mathbf{u}=\lambda \mathbf{u}$ and $\mathbf{M}^{q} \mathbf{v}=b^{q} \mathbf{v}=\lambda \mathbf{v}$. As $q$ is odd, this means $a=b$ and, consequently, $\mathbf{M}=\lambda^{\frac{1}{q}} \mathbf{I}_{2}$.

Now, assume there exists a cycle with $q \geq 2$ with $q$ even. From (15) and as $\lambda>0, \frac{1}{\sqrt{\lambda}} \mathbf{M}^{\frac{q}{2}}$ is its own inverse which implies that the eigenvalues of $\mathbf{M}^{\frac{q}{2}}$ are $\pm \sqrt{\lambda}$.

Let us assume $\frac{q}{2}$ is odd. If the eigenvalues of $\mathbf{M}^{\frac{q}{2}}$ are equal then $\mathbf{M}=\lambda^{\frac{1}{q}} \mathbf{I}_{2}$. Otherwise if $\mathbf{u}$ and $\mathbf{v}$ are eigenvectors of $\mathbf{M}$ associated with $a$ and $b$ respectively, then we $a^{\frac{q}{2}}=-b^{\frac{q}{2}}$, (the relation is obtained by applying $\mathbf{M} \frac{q}{2}$ times), leading to $a=-b$. Thus, when $\mathbf{M}$ is not proportional to $\mathbf{I}_{2}$, it is similar to:

$$
\mathbf{M}_{\text {sim }}=\left(\begin{array}{cc}
\lambda^{\frac{1}{q}} & 0 \\
0 & -\lambda^{\frac{1}{q}}
\end{array}\right) \text {. }
$$

When $\frac{q}{2}$ is even, $\mathbf{M}^{\frac{q}{2}}$ has a unique eigenvalue $\sqrt{\lambda}$ (we recall that the eigenvalues of $\mathbf{M}$ are real); that is $\mathbf{M}^{\frac{q}{2}}=\sqrt{\lambda} \mathbf{I}_{2}$. Applying the same reasoning as previously we obtain that every matrix $\mathbf{M}$ that is not of the form $a \mathbf{I}_{2}$ and that satisfy property (4) is similar to $\mathbf{M}_{\text {sim }}$, defined in (16).

We deduce that any matrix $\mathbf{M}$ that is worth studying has a null trace, which leads to $\mathbf{M}^{2}=\lambda \mathbf{I}_{2}$.

Let us now give two illustrations: the first one is the quincunx sampling matrix and the second is the hexagonal sampling matrix. The quincunx sampling matrix is defined by [14]

$$
\mathbf{M}=\left(\begin{array}{cc}
-1 & 1 \\
1 & 1
\end{array}\right)
$$

Here, $\mathbf{M}^{2}=2 \mathbf{I}_{2}$ and $\mathbf{M}$ is thus similar to

$$
\left(\begin{array}{cc}
\sqrt{2} & 0 \\
0 & -\sqrt{2}
\end{array}\right)
$$

The hexagonal sampling matrix is

$$
\mathbf{M}=\left(\begin{array}{cc}
2 & 1 \\
0 & -2
\end{array}\right) .
$$

As $\mathbf{M}^{2}=4 \mathbf{I}_{2}, \lambda=4$ leading to $\mathbf{M}$ is similar to

$$
\left(\begin{array}{cc}
2 & 0 \\
0 & -2
\end{array}\right) \text {. }
$$




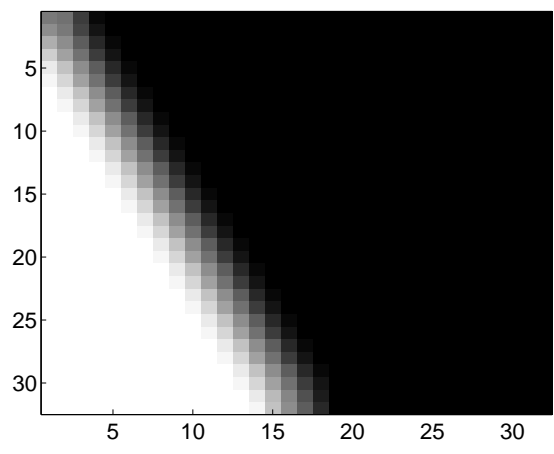

(A)

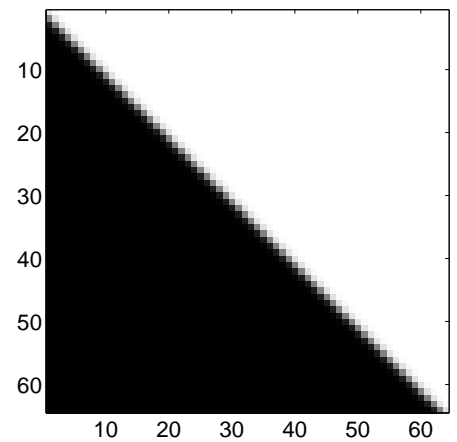

(C)

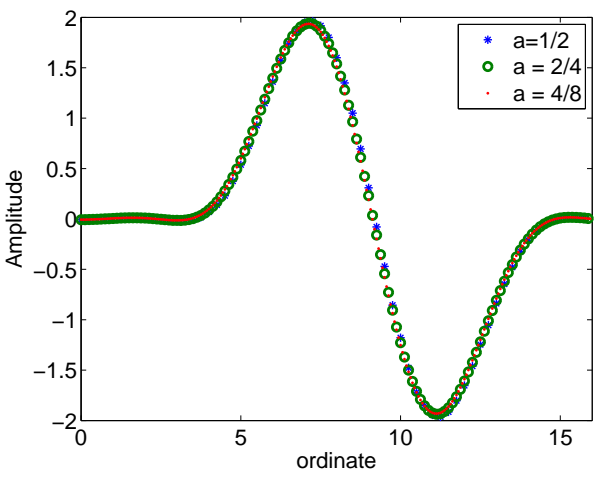

(B)

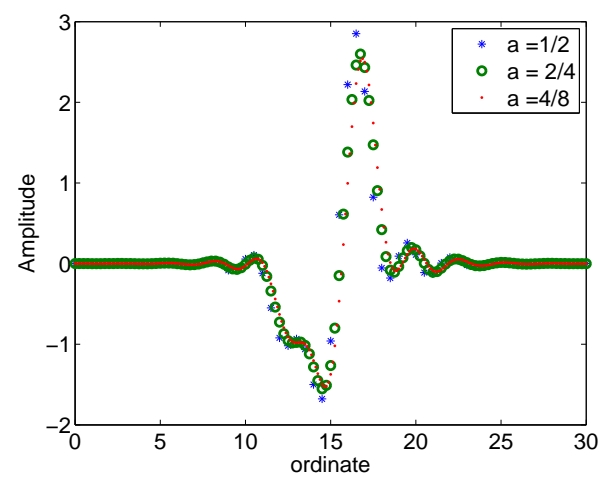

(D)

Fig. 1. (A): $32 \times 32$ step-edge image (amplitude of the step-edge $=2$ ) with support $\{0, \cdots, 31\} \times\{0, \cdots, 31\}$, the direction of the edge being the vector $\left[\begin{array}{ll}2 & 1\end{array}\right]^{T}$; (B): plot of the scale-space filtering, defined by the hexagonal sampling matrix with $m=1$ and applied to image (A), in the direction $\left[\begin{array}{ll}1 & -2\end{array}\right]^{T}$ starting from the initial coordinates $(20,0)$ with respect to the ordinate. We consider $a=\frac{1}{2}$ for $m_{1}=1, m_{2}=2$ or $m_{1}=2, m_{2}=4$ or $m_{1}=4, m_{2}=8$ (C): diagonal $64 \times 64$ step-edge image (amplitude of the step edge $=2$ ), with support $\{0, \cdots, 63\} \times\{0, \cdots, 63\}$; (D): plot of the scale-space filtering, defined by the quincunx sampling matrix with $m=1$ and applied to image (C), in the direction $\left[\begin{array}{ll}-1 & 1\end{array}\right]^{T}$ starting from the initial coordinates $(32,0)$ with respect to the ordinate. We consider $a=\frac{1}{2}$ for $m_{1}=1, m_{2}=2$, or $m_{1}=2, m_{2}=4$ or $m_{1}=4, m_{2}=8$

\section{B. Invariant Sets of Four Vectors}

To apply Theorem 3, we need a set $\mathbf{X}_{n}$ such that $\left\{\mathbf{x}_{1}, \cdots, \mathbf{x}_{i}^{*}, \cdots, \mathbf{x}_{n}\right\}$ spans $\mathbb{R}^{2}$ and we also require that $\beta$ be continuously differentiable. When $\mathbf{X}_{n}$ is made of non-collinear vectors, the latter condition imposes that $\mathbf{X}_{n}$ contains at least 4 vectors. Indeed, $\beta$ is in $C^{r}\left(\mathbb{R}^{2}\right)$ with

$$
r=\min \left\{\# Y: Y \subset \mathbf{X}_{n}, \mathbf{X}_{n} \backslash Y \neq \mathbb{R}^{2}\right\}-2,
$$


where $\# Y$ stands for the cardinal of $Y$ [5]. We now assume that $\mathbf{X}_{n}$ is composed of 4 non-collinear vectors. $\mathbf{X}_{n}$ must also be such that property (4) is satisfied. We now show, given $\mathbf{M}$, how to define such a vector set.

As $\mathbf{M}^{2}=\lambda \mathbf{I}_{2}$, with $\lambda>0$, for any vector $\mathbf{u}$ which is not an eigenvector of $\mathbf{M}$, if we put $\mathbf{M u}=\mathbf{v}$, we necessarily have $\mathbf{M v}=\lambda \mathbf{u}$. Therefore, to define a 4 invariant vectors set, it suffices to consider two vectors that are not eigenvectors of $\mathbf{M}$. Let us consider the set $\left\{\left[\begin{array}{ll}1 & 0\end{array}\right]^{T},\left[\begin{array}{ll}0 & 1\end{array}\right]^{T},\left[\begin{array}{ll}1 & 1\end{array}\right]^{T},\left[\begin{array}{ll}-1 & 1\end{array}\right]^{T}\right\}$. For any matrix $\mathbf{M}$ at least two of these vectors are not eigenvectors. We consider the first two which are not eigenvectors, to build the invariant set. For instance, for the quincunx matrix, $\left[\begin{array}{ll}1 & 0\end{array}\right]^{T}$ and $\left[\begin{array}{ll}0 & 1\end{array}\right]^{T}$ are not eigenvectors leading to the invariant vector set $\mathbf{X}_{n}=\left\{\left[\begin{array}{ll}1 & 0\end{array}\right]^{T},\left[\begin{array}{ll}-1 & 1\end{array}\right]^{T},\left[\begin{array}{ll}0 & 1\end{array}\right]^{T},\left[\begin{array}{ll}1 & 1\end{array}\right]^{T}\right\}$. Similarly, for the hexagonal sampling, $\left[\begin{array}{ll}0 & 1\end{array}\right]^{T}$ and $\left[\begin{array}{ll}1 & 1\end{array}\right]^{T}$ are not eigenvectors leading to $\mathbf{X}_{n}=\left\{\left[\begin{array}{ll}0 & 1\end{array}\right]^{T},\left[\begin{array}{ll}1 & -2\end{array}\right]^{T},\left[\begin{array}{ll}1 & 1\end{array}\right]^{T},\left[\begin{array}{ll}3 & -2\end{array}\right]^{T}\right\}$.

\section{NumericAl APPLiCATIONS}

We now study the behavior of the scale-space filtering we propose. In image processing, it is usual to consider that the first component of a vector (i.e. abscissa) is the coordinate on the vertical axe oriented downward while the second component (i.e ordinate) is the coordinate on the horizontal axe oriented to the right. This convention holds for the rest of the paper. For image boundary conditions, we adopt mirror conditions that is we symmetrize the image with respect to its boundary [7]. In the following two subsections, we aim at showing that to choose an appropriate sampling matrix leads to a better resolution of the scale-space filtering in the direction orthogonal to the edge and, second, that the convergence of the approximation (13) to the true value (12) is very fast. As each sampling matrix $\mathbf{M}$ is such that $\mathbf{M}^{2}$ is proportional to $\mathbf{I}_{2}, \mathbf{M}^{-m}, m$ odd is proportional to $\mathbf{M}^{-1}$, therefore it is essentially worth studying the approximation (13) when $m=1$.

\section{A. Scale-Space Filtering on Step-Edge Images}

To illustrate the interest of using the hexagonal sampling matrix, we first consider a step-edge image as that of Figure 1.A which is such that the vector $\left[\begin{array}{ll}1 & -2\end{array}\right]^{T}$ is orthogonal to the step-edge. When $\mathbf{M}$ is the hexagonal sampling matrix, computing the scale-space filtering (13), for $m \neq 0$ when $m=1$ leads to a four times finer resolution in the direction orthogonal to the edge is four times finer than that with $m=0$. For the present example, we thus consider that $\mathbf{M}$ is the hexagonal sampling matrix, $\mathbf{X}_{n}=\left\{\left[\begin{array}{ll}0 & 1\end{array}\right]^{T},\left[\begin{array}{ll}1 & -2\end{array}\right]^{T},\left[\begin{array}{ll}1 & 1\end{array}\right]^{T},\left[\begin{array}{ll}3 & -2\end{array}\right]^{T}\right\}$ and $m=1$. The wavelet $\Psi$ we use here is designed to study the singularities in the direction of $\mathbf{x}_{2}$; it is defined by (14) with $\alpha$ corresponding to the convolution of 
$\left(\tilde{\alpha}_{i}\right)_{1 \leq i \leq 4}$ that is:

$$
\alpha=\frac{\tilde{\alpha}_{1} * \tilde{\alpha}_{2} * \tilde{\alpha}_{3} * \tilde{\alpha}_{4}}{\max _{\mathbf{p} \in \mathbb{Z}^{2}} \sum_{\mathbf{k} \in \mathbb{Z}^{2}}\left(\tilde{\alpha}_{1} * \tilde{\alpha}_{2} * \tilde{\alpha}_{3} * \tilde{\alpha}_{4}\right)[\mathbf{k}] \beta\left(\mathbf{p}-\mathbf{k}, \mathbf{X}_{n}\right)}
$$

with $\tilde{\alpha}_{2}\left[-\mathbf{x}_{2}\right]=-1, \tilde{\alpha}_{4}[0]=2, \tilde{\alpha}_{2}\left[\mathbf{x}_{2}\right]=-1$ and zero elsewhere and, for $j \neq 2, \tilde{\alpha}_{j}\left[-\mathbf{x}_{j}\right]=1, \tilde{\alpha}_{j}[0]=2$, $\tilde{\alpha}_{j}\left[\mathbf{x}_{j}\right]=1$ and zero elsewhere. The wavelet thus defined is such that its value at integer locations is less than one. One can also check that $(\alpha[\mathbf{p}])_{\mathbf{p} \in \mathbb{Z}^{2}}$ sums to zero.

We apply the scale-space filtering defined by (13) to the step-edge image of Figure 1.A, whose support is $\{0, \cdots, 31\} \times\{0, \cdots, 31\}$. We consider $a=\frac{m_{1}}{m_{2}}=\frac{1}{2}$, either with $m_{1}=1, m_{2}=2$, or $m_{1}=2, m_{2}=4$, or $m_{1}=4, m_{2}=8$. We display, in Figure 1.B, the coefficients $W \tilde{f}_{a}$ in the direction of the vector $\left[\begin{array}{ll}2 & 1\end{array}\right]^{T}$ with respect to the ordinate and starting from the point with coordinates $(20,0)$. We do not display the exact coefficients $W \tilde{f}$ since when $m_{1}=4$ and $m_{2}=8, W \tilde{f}$ and $W \tilde{f}_{a}$ are visually indistinguishable.

We then consider a diagonal step-edge function as that of Figure 1.C, whose support is $\{0, \cdots, 63\} \times$ $\{0, \cdots, 63\}$. When $m=1$ and the quincunx sampling matrix is used, the resolution of the scale-space filtering is two times finer in the direction orthogonal to the edge than when $m=0$. We therefore implement the scale-space filtering with $\mathbf{M}$ the quincunx sampling matrix and $\mathbf{X}_{n}=\left\{\left[\begin{array}{ll}1 & 0\end{array}\right]^{T},\left[\begin{array}{ll}-1 & 1\end{array}\right]^{T},\left[\begin{array}{ll}0 & 1\end{array}\right]^{T},\left[\begin{array}{ll}1 & 1\end{array}\right]^{T}\right\}$. The wavelet $\Psi$ we use is designed to study the singularities in the direction of $\mathbf{x}_{2}$. The filter $\alpha$ is thus built as previously, taking into account the change of vector set $\mathbf{X}_{n}$. We display in Figure 1.D, the coefficients $W \tilde{f}_{a}$ in the direction of the vector $\left[\begin{array}{ll}-1 & 1\end{array}\right]^{T}$ with respect to the ordinate and starting from the point with coordinates $(32,0)$. Again, $a=\frac{m_{1}}{m_{2}}=\frac{1}{2}$ either with $m_{1}=1, m_{2}=2$, or $m_{1}=2, m_{2}=4$ or $m_{1}=4, m_{2}=8$. We do not display the exact coefficient given by (12) since when $m_{1}=4, m_{2}=8$ the approximation and the exact scale-space filtering are visually indistinguishable.

Of course, different edge orientations would require different sampling matrices $\mathbf{M}$ but this will be the topic for future work.

\section{B. An Illustrative Example on a Natural Image}

We now apply the scale-space filtering we propose to the image of Lenna. In this illustrative example, we consider that $\mathbf{X}_{n}$ is associated to the quincunx sampling, that the wavelet is designed to study the singularities in the direction $\mathbf{x}_{1}=\left[\begin{array}{ll}1 & 0\end{array}\right]^{T}$ following the same framework as in the previous subsection and also that $m=0$. We furthermore consider $a=\frac{m_{1}}{m_{2}}=\frac{1}{2}$. As already pointed out, taking $m_{1}=4$ and $m_{2}=8$ provides an approximation of the scale-space filtering which is visually indistinguishable from the true value. We display in Figure 2.A, the original image of Lenna and then in Figure 2.B-D, the approximation of the scale-space filtering for a region of the eye, of the fur and of the hat respectively 


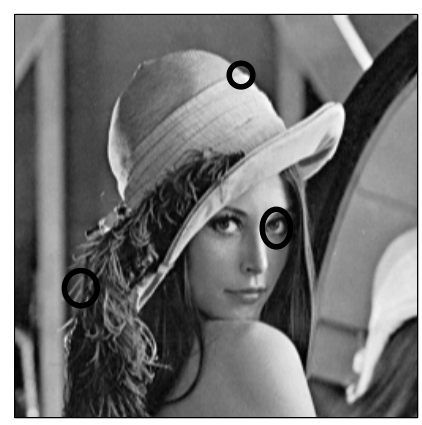

(A)

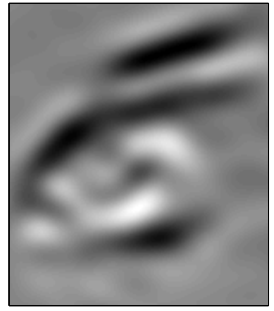

(B)

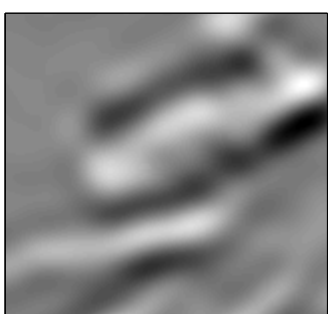

(C)

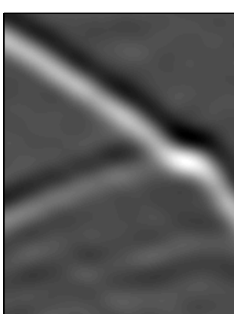

(D)

Fig. 2. (A): image of Lenna ; (B): scale-space filtering for the region of the eye when $m_{1}=4$ and $m_{2}=8$; (C): similar computation to (B) for a region of the fur; (D): similar computation to $(\mathrm{C})$ for a region of the hat.

corresponding to the surrounded regions in Figure 2.A. Through these examples, we notice that the scale space filtering we propose can both handle geometric and textural parts of images, and that, as expected, the singularities in the vertical direction (i.e. the direction of $\mathbf{x}_{1}$ ) are well detected and can be precisely localized, since they correspond to the coefficients with the largest amplitudes (black or white pixels) in Figure 2.B-D.

\section{CONCLUSION}

In this paper, we have introduced a new scale-space filtering based on a box spline representation of multidimensional signals. The scale-space filtering is computed at rational scales and at locations dependent on some sampling matrix and on a dilation factor $\left(m_{2}\right.$ in the paper). Using box splines is interesting in that it enables the computation of the scale-space filtering on a non Cartesian grid. In particular, this allows a better localization of the edges at finer scales without increasing the computational cost. We have also shown that we can save computation time using an approximation of the scale-space 
filtering based on box splines. This approximation does not generate any visual distortion provided the length of the filters used are large enough. In practice, these may be chosen with a relatively small size ; this aspect is important to preserve a low computational cost for the proposed method. In a near future, we will investigate more in detail the relation between the sampling matrix and edge orientation and wether it is possible to build a scale-space filtering involving box splines associated with different sampling matrices.

\section{APPENDIX}

\section{A. Proof of Theorem 1}

We first recall that the Fourier transform of $\beta\left(\mathbf{x}, \mathbf{X}_{n}\right)$ is [3]

$$
\mathcal{F}(\beta)\left(\chi, \mathbf{X}_{n}\right)=\prod_{p=1}^{n} \frac{1-e^{-i \chi^{T} \mathbf{x}_{p}}}{i \chi^{T} \mathbf{x}_{p}}
$$

Then, using property (4), we can write

$$
\frac{\mathcal{F}(\beta)\left(\mathbf{M}^{T} \chi, \mathbf{X}_{n}\right)}{\mathcal{F}(\beta)\left(\chi, \mathbf{X}_{n}\right)}=\prod_{p=1}^{n} \frac{1}{\lambda_{p}} \frac{1-e^{-i \lambda_{p} \chi^{T} \mathbf{x}_{\sigma(p)}}}{1-e^{-i \chi^{T} \mathbf{x}_{\sigma(p)}}}=\frac{1}{\sqrt{|\operatorname{det}(\mathbf{M})|}} G\left(\chi, \mathbf{X}_{n}\right) .
$$

Equation (17) then can be written as follows:

$$
\mathcal{F}(\beta)\left(\mathbf{M}^{T} \chi, \mathbf{X}_{n}\right)=\prod_{p=1}^{n} \frac{1}{\lambda_{p}}\left(1+e^{-i \chi^{T} \mathbf{x}_{\sigma(p)}}+\cdots+e^{-i\left(\lambda_{p}-1\right) \chi^{T} \mathbf{x}_{\sigma(p)}}\right) \mathcal{F}(\beta)\left(\chi, \mathbf{X}_{n}\right) .
$$

Let us now recall some basic properties of the Fourier transform. If $(h[\mathbf{n}])_{\mathbf{n} \in \mathbb{Z}^{s}}$ is a finite sequence, for an integrable function $f$ defined on $\mathbb{R}^{s}$, then $\mathcal{F}\left(\sum_{\mathbf{k} \in \mathbb{Z}^{s}} h[\mathbf{k}] f(.-\mathbf{k})\right)(\chi)=\hat{\mathbf{h}}(\chi) \mathcal{F}(\mathbf{f})(\chi)$, where $\hat{h}(\chi)=\sum_{\mathbf{n} \in \mathbb{Z}^{\mathbf{s}}} \mathbf{h}[\mathbf{n}] \mathbf{e}^{-\mathbf{2 i} \pi<\mathbf{n}, \chi>}$, with $<\cdot, \cdot>$ being the Euclidean inner product on $\mathbb{R}^{s}$. From this we deduce

$$
\mathcal{F}(\beta)\left(\chi, \mathbf{X}_{n}\right)=\mathcal{F}\left(\sqrt{|\operatorname{det}(\mathbf{M})|} \sum_{\mathbf{p} \in \mathbb{Z}^{s}} g\left[\mathbf{p}, \Lambda_{n}, \mathbf{X}_{\sigma(n)}\right] \beta\left(\mathbf{M x}-\mathbf{p}, \mathbf{X}_{n}\right)\right)(\chi),
$$

for all $\mathbf{x}$ in $\mathbb{R}^{s}$ where

$$
g\left[\mathbf{p}, \Lambda_{n}, \mathbf{X}_{n}\right]=\frac{\sqrt{|\operatorname{det}(\mathbf{M})|}}{\prod_{p=1}^{n} \lambda_{p}}\left(b_{\lambda_{1}}\left[., \mathbf{x}_{1}\right] * \cdots * b_{\lambda_{n}}\left[., \mathbf{x}_{n}\right]\right)[\mathbf{p}] .
$$

Both terms in equation (18) are in $L^{2}\left(\mathbb{R}^{s}\right)$, so that $\beta\left(\mathbf{x}, \mathbf{X}_{n}\right)=\sqrt{|\operatorname{det}(\mathbf{M})|} \sum_{\mathbf{p} \in \mathbb{Z}^{s}} g\left[\mathbf{p}, \Lambda_{n}, \mathbf{X}_{\sigma(n)}\right] \beta(\mathbf{M x}-$ $\left.\mathbf{p}, \mathbf{X}_{n}\right)$, almost everywhere. Since each term in the equation is continuous, the equality holds for all $\mathbf{x}$. 


\section{B. Proof of Theorem 2}

For any $m \in \mathbb{N}$ and using the definition of $G$ given in the proof of Theorem 1, we have:

$$
\begin{aligned}
\frac{\mathcal{F}(\beta)\left(\left(\mathbf{M}^{T}\right)^{m} \chi, \mathbf{X}_{n}\right)}{\mathcal{F}(\beta)\left(\chi, \mathbf{X}_{n}\right)}= & \prod_{r=1}^{m} \frac{\mathcal{F}(\beta)\left(\left(\mathbf{M}^{T}\right)^{r} \chi, \mathbf{X}_{n}\right)}{\mathcal{F}(\beta)\left(\left(\mathbf{M}^{T}\right)^{r-1} \chi, \mathbf{X}_{n}\right)}=\prod_{r=1}^{m} \frac{1}{\sqrt{|\operatorname{det}(\mathbf{M})|}} G\left(\left(\mathbf{M}^{T}\right)^{r-1} \chi, \mathbf{X}_{n}\right) \\
& =\prod_{r=1}^{m} \prod_{p=1}^{n} \frac{1}{\lambda_{p}}\left(1+e^{-i \chi^{T} \mathbf{M}^{r-1} \mathbf{x}_{\sigma(p)}}+\cdots+e^{-i\left(\lambda_{p}-1\right) \chi^{T} \mathbf{M}^{r-1} \mathbf{x}_{\sigma(p)}}\right) .
\end{aligned}
$$

As for $r \geq 2, \mathbf{M}^{r-1} \mathbf{x}_{\sigma(p)}=\prod_{k=1}^{r-1} \lambda_{\sigma^{k}(p)} \mathbf{x}_{\sigma^{r}(p)}$ and using the same properties of the Fourier transform as in the proof of Theorem 1, we can write the following equation:

$$
\beta\left(\mathbf{x}, \mathbf{X}_{n}\right)=\sqrt{|\operatorname{det}(\mathbf{M})|^{m}} \sum_{\mathbf{p} \in \mathbb{Z}^{s}} g_{m}\left[\mathbf{p}, \Lambda_{n}, \mathbf{X}_{n}\right] \beta\left(\mathbf{M}^{m} \mathbf{x}-\mathbf{p}, \mathbf{X}_{n}\right),
$$

with

$$
g_{m}\left[\mathbf{p}, \Lambda_{n}, \mathbf{X}_{n}\right]=\left(\tilde{g}\left[., \Lambda_{n}, \mathbf{X}_{\sigma(n)}\right] * \cdots * \tilde{g}\left[., \Lambda_{n}, \mathbf{X}_{\sigma^{m}(n)}\right]\right)[\mathbf{p}],
$$

where $\tilde{g}\left[\mathbf{p}, \Lambda_{n}, \mathbf{X}_{\sigma(n)}\right]=g\left[\mathbf{p}, \Lambda_{n}, \mathbf{X}_{\sigma(n)}\right]$ and for $r \geq 2$,

$$
\tilde{g}\left[\mathbf{p}, \Lambda_{n}, \mathbf{X}_{\sigma^{r}(n)}\right]=\frac{\sqrt{|\operatorname{det}(\mathbf{M})|}}{\prod_{p=1}^{n} \lambda_{p}}\left(\left(b_{\lambda_{1}}\right) \uparrow_{k=1}^{r-1} \lambda_{\sigma^{k}(1)}\left[., \mathbf{x}_{\sigma^{r}(1)}\right] * \cdots *\left(b_{\lambda_{n}}\right) \underset{\uparrow \prod_{k=1}^{r-1} \lambda_{\sigma^{k}(n)}}{ }\left[., \mathbf{x}_{\sigma^{r}(n)}\right]\right)[\mathbf{p}] .
$$

\section{REFERENCES}

[1] T. Asahi, K. Ichige, and R. Ishii, An efficient Algorithm for Decomposition and Reconstruction of Images by Box Splines, IEICE Transactions on Fundamentals, vol. E84-A,no. 8, pp. 1883-1891, 2001.

[2] T. Asahi, K. Ichige and R. Ishii, A New Formulation for Discrete Box Splines Reducing Computational Cost and its Evaluation, IEICE Transactions on Fundamentals, vol. E84-1,no. 3, pp. 884-892, 2001.

[3] C. K. Chui, Multivariate Splines, SIAM, Philadelphia, Pennsylvania, 1988.

[4] W. Dahmen, C.A. Michelli, On the Local Linear Independence of Translates of Box Splines Studia Math.,vol; 82, pp.243-262, 1985.

[5] C. De Boor, K. Höllig, B-Splines from parallelepipeds, J. Analyse Mathématique, vol. 42, pp.99-115, 1982.

[6] C. De Boor, K. Höllig and S. Riemenschneider, Box Splines, Applied Mathematical Sciences 98, Springer, New York, 1993.

[7] H. Kiya, K. Nishikawa and Iwahashi, A development of symmetric extension method for subband image coding, IEEE Transactions on Image Processing, vol. 3, no. 1, pp. 78-81, 1994.

[8] S. Malassiotis and M.G. Strintzis, Optimal Biorthogonal Wavelet Decomposition of Wire-Frame Meshes Using Box Splines, and its Application to the Hierarchical Coding of 3-D Surfaces IEEE Transactions on Image Processing, vol.8,no.1, pp. 41-57, 1999.

[9] S. Meignen, Application of the Convergence of the Control Points of B-Splines to Wavelet Decomposition at Rational Scales and Rational Location, IEEE Signal Processing Letters, vol. 12, no. 1, pp. 29-32, 2005.

[10] H. Prautsch, W. Boehm, M. Paluszny, Bézier and B-spline Techniques, Springer, 2002. 
[11] M. G. Strintzis, Optimal Biorthogonal Wavelet Bases for Signal Decomposition IEEE Transactions on Signal Processing, vol. 44, no. 6, pp. 1406-1417, 1996.

[12] J. Stöckler, Multivarite Wavelets, in Wavelets: a Tutorial in Theory and Applications, C.K. Chui, Ed. New York: Academic , 1992, pp. 325-355.

[13] M. Unser, A. Aldroubi and M. Eden, Fast B-spline Transform for Continuous Image Representation and Interpolation, IEEE Transactions on Pattern Analysis and Machine Intelligence, vol.13, no.3, pp. 277-285, 1991.

[14] P.P. Vaidyanathan, Multirate Systems and Filter Banks, Englewood Cliffs,NJ: Prentice-Hall, 1993.

[15] Y.P. Wang and S.L. Lee, Scale-Space Derived from B-Splines, IEEE Transactions on Pattern Analysis and Machine Intelligence, vol. 20, no. 10, pp. 1040-1055,1998.

[16] R. A. Young, The Gaussian Derivative Model for Machine Vision: Visual Cortex Simulation, J. Optical. Soc. Am., July 1987. 